\title{
ELEVANDO A AUTOESTIMA: A ASSOCIAÇÃO DO COSMÉTICO COMO RECURSO TERAPÊUTICO PARA MULHERES EM TRATAMENTO ONCOLÓGICO
}

\author{
RAISING SELF-ESTEEM: THE ASSOCIATION OF THE COSMETIC AS A \\ THERAPEUTIC RESOURCE FOR WOMEN UNDER ONCOLOGICAL TREATMENT
}

\author{
Mônica Santos ${ }^{1}$, Ana Paula L. Amorim¹, Airton Rodrigues²
}

1 - Graduanda do Curso de Tecnologia em Cosméticos, da Faculdade de Tecnologia de Diadema Luigi Papaiz.

2 - Professor Doutor. Docente da Faculdade de Tecnologia de Diadema Luigi Papaiz.

\section{RESUMO:}

Introdução: As pessoas buscam os cosméticos para melhorar a autoestima. Os efeitos deste bem-estar psicológico podem ser vistos como forma terapêutica junto a determinados grupos de pacientes, especificamente oncológicos. O aumento na autoestima proporciona um estado de espírito mais confiante para enfrentar as adversidades da doença. Objetivo: Associar o uso de cosmético como um recurso viável de terapia e promoção da autoestima buscando compreender a sua relação com a saúde mental, embora subdimensionado pela comunidade cientifica. Material e métodos: Através da revisão narrativa, pesquisou-se sobre o tema em artigos, relatórios, reportagens e entrevista concedida pelo Instituto ABIHPEC com o gestor do programa "De Bem Com Você - A Beleza Contra o Câncer". Resultados e Discussão: Ao experimentar programas que visam promover a autoestima das mulheres em tratamento da doença, muitas participantes apresentaram um maior bemestar e confiança relacionado a sua aparência. Os tratamentos de beleza são uma terapia adjuvante aos tratamentos oncológicos trazendo profundo impacto positivo às mulheres. Considerações Finais: $O$ uso de cosméticos como recurso terapêutico contribui positivamente na elevação da autoestima de mulheres em tratamento oncológico sendo este uma ferramenta de apoio. Contudo, existe uma carência de estudos relacionados ao tema oportunizando novas pesquisas nesta área da ciência.

Palavras Chaves: Cosmético, mulher, autoestima, tratamento, câncer.

\begin{abstract}
:
Introduction: People seek cosmetics to boost self-esteem. The effects of this psychological welfare can be seen as a therapeutic way combined with certain groups of patients, especially oncological ones. The boost in self-esteem provides a more confidente state of mind to face the adversities of the disease. Objective: Associate the use of cosmetics as a viable resource of therapy and the promotion of self-esteem, trying to understand its relation with mental health, although the topic is still undermined by the scientfic community. Material and Methods: Through the narrative review, the topic was reaserched in articles, reports, surveys and interviews conceded by the Institute ABIHPEC with the program manager "De Bem Com Você - A Beleza Contra o Cancêr". Results and Discussion: After experimenting programs which aim to promote women's self-esteem during treatment, many of the participants present higher self-esteem and confidence towards their appearance. The beauty treatments are a therapy alongside oncological treatments, bringing a deeper positive impact on women. Final Considerations: The use of cosmetics as a therapeutic resource contributed positively to the rise of self-esteem in women in oncological treatment,
\end{abstract}


as a support tool. However, there's a scarcity of studies in the subject, preventing new researched on the area.

Key-Words: Cosmetics; Woman; Self-Esteem; Treatment; Cancer.

\section{INTRODUÇÃO}

De acordo com a Organização Mundial da Saúde (OMS), "saúde é um estado de completo bem-estar físico, mental e social e não apenas a ausência da doença". Refletindo sobre esta definição de saúde, os cosméticos remetem como parte da qualidade de vida, vaidade e autoestima (OPAS, 2020). Transcendendo uma visão de que a saúde não está ligada apenas a distúrbios físicos (SILVA, 2018).

A vaidade pode ser entendida pela relação do status social junto com a autoestima do indivíduo (FAN, 2014). Os conceitos de vaidade são sustentados por dois pilares, o primeiro no que tange a questão física, pode conduzir a uma visão positiva e possivelmente exagerada da aparência, e o segundo, na forma de realização pessoal, guiado por uma preocupação excessiva e / ou uma visão positiva e quiçá até inflada de realizações pessoais (NETEMEYER; BURTON; LICHTENSTEIN, 1995).

O conceito de autoestima tem sido estudado e conduzido como um significante indicador de saúde mental (FLORIANI; MARCANTE, 2010). Partindo-se da ideia de que a autoestima é uma necessidade humana que contribui para o processo da vida, quando positiva funciona como o sistema imunológico da consciência, entretando, quando baixa esse sistema torna-se vulnerável para enfrentar as adversidades da vida interditando 0 crescimento psicológico do indivíduo (BORBA, 2011).

Para que tal situação não ocorra, a autoestima deve ser consolidada como uma imagem positiva de si próprio, é ter a capacidade de vislumbrar a beleza e as qualidades sem expor os defeitos, evitar comparações já que cada pessoa tem uma beleza única, a estética não pode ser padronizada e a autoestima deve ser fortalecida para que o indivíduo tenha uma vida de qualidade, felicidade e autoconfiança (BORBA, 2011).

A aplicação de cosméticos no corpo humano tem função de limpeza e embelezamento, promovendo atratividade ou alterando a aparência, sem afetar a estrutura ou as funções do corpo (MOTA et al., 2014).

É sabido que com o passar do tempo, as pessoas buscam cosméticos no intuito de elevar e melhorar a autoestima e seu bem-estar, sendo disposto pelo mercado diversos produtos e serviços com esta finalidade. Estudos indicam que a vaidade está por trás de autoestima (AVELAR; VEIGA, 2013). Apontando que indivíduos vaidosos tendem a se 
enquadrar em um padrão estético de autovalorização, bem-estar e alta autoestima (LYUBOMIRSKY; ROSS, 1997). Já os indivíduos menos vaidosos, podem criar insatisfação e se sentir desconfortáveis com o próprio corpo e com a autoestima baixa, criando uma espécie de armadilha (GOMES; CARAMASCHI, 2007).

Quanto maior o grau de vaidade da mulher maior a relação entre sua autoestima com a aparência e o corpo (STREHLAU; CLARO; NETO, 2010). Para mulheres em enfrentamento ao câncer, programas são criados a fim de amparar e fornecer recursos para que este panorama não seja ainda mais abalado pelos efeitos deletérios do tratamento na aparência (AVELAR; VEIGA, 2013).

O Look Good Feel Better (LGFB) consiste em um programa de apoio social, neutro e não médico que ensina técnicas de beleza a pessoas com câncer, teve seu início em 1989 nos Estados Unidos, pela organização de caridade Look Good Feel Better Foundation, entretanto, sua origem se deu com um médico ao observar que uma paciente de câncer mal saía de seu quarto devido aos efeitos colaterais causados pelo tratamento na sua aparência, ele então pediu ajuda ao ex-presidente do Conselho de Produtos de Cuidado Pessoal, Ed Kavanaugh, que por sua vez providenciou cosméticos e um cosmetologista transformando não apenas o visual da mulher, mas também sua aparência, arrancando-lhe um sorriso do rosto e proporcionando-Ihe um bem-estar que a tempos não sentia. A partir daí, Kavanaugh reconheceu a oportunidade de benfeitoria e apresentou a ideia aos membros do Conselho de Produtos de Cuidado Pessoal - os líderes da indústria de cosméticos do país que abraçaram a causa de ajudar mais mulheres a manter sua confiança e autoestima (BETTER, 2020).

Além do programa dos EUA, que atende mais de 60mil mulheres por ano, a Fundação Look Good Feel Better supervisiona uma rede global de 26 afiliados licenciados que oferecem programas de apoio LGFB em países de todo o mundo. Juntos, dezenas de milhares de voluntários ajudaram mais de 2 milhões de pessoas que vivem com câncer em seis continentes desde o início do programa em 1989. O programa inclui aulas de cuidados com a pele e unhas, cosméticos, perucas e turbantes, acessórios e styling, ajudando as pessoas com câncer a encontrarem um pouco de normalidade em uma vida que de forma alguma é normal (BETTER, 2020).

As reuniões LGFB são chamadas de 'workshops de transformação em grupo' em que cada participante recebe um necessaire com produtos cosméticos identificados com os tons claro, médio e escuro pra combinar com o tom de pele das participantes. No processo, os medos são diminuídos, amizades são formadas e a autoestima e o senso de 
normalidade começam a ressurgir. Para as mulheres que participam, os benefícios são significativos (ROARK, 2008). Uma vez que aprendem a disfarçar as evidências físicas provenientes dos tratamentos contra o câncer, deixando-a mais autoconfiante durante 0 processo (KENDRICK, 2008). Após a entrega um vídeo é transmitido no qual mulheres são mostradas participando de uma reunião e dando depoimentos sobre seu histórico de câncer e o valor que o programa tem, passando a mensagem de que as mulheres devem estar bem ao passar pelo tratamento do câncer porque isso as ajudará a "esconder" a doença e a aumentar sua autoestima pelo incentivo do uso de cosméticos, visto que os tratamentos de quimioterapia e radioterapia sensibilizam sua aparência física. Profissionais de beleza voluntários lideram o workshop com ensinamentos de técnicas de maquiagem, cuidados com a pele e instruções de como lidar com a queda de cabelo. O programa também traz orientações para jogar fora os cosméticos velhos porque podem conter bactérias e os tratamentos do câncer suprimem o sistema imunológico. Os participantes também são incentivados a ajudar uns aos outros a se maquiar e compartilham dicas de saúde ou beleza que possam ter (KENDRICK, 2008).

Evidências apontam que a autoestima possui efeitos positivos sobre o bem-estar. Sob outra perspectiva, conectado a autoestima, estão os cosméticos, cuja principal função é reconstruir a autoimagem. Este trabalho tem como propósito ligar dois elos desta corrente: a autoestima e os cosméticos como fator terapêutico.

\section{FUNDAMENTAÇÃO TEÓRICA}

\subsection{Autoestima relacionada a doença}

Para pacientes vítimas de queimaduras a manutenção da autoestima se revela ser o principal fator na reabilitação social (LAPORTE; LEONARDI, 2010). Lesões de pele como eritema, acne, melasma, lúpus discoides, hiperpigmentação, vitiligo, xantelasma, manchas do vinho do porto, cicatrizes e marcas de nascença estão entre os tipos de lesões que mais acometem a pele e a maquiagem permite camuflar estas deformidades e melhorar a qualidade de vida através do uso de produtos com textura mais densa do que os normais que buscam cobrir perfeitamente as imperfeições da pele (OLIVEIRA; ARAÚJO; FERNANDES, 2012).

Receber o diagnóstico de um câncer é muito doloroso e os tratamentos não atingem apenas a esfera física da vida da paciente, mas também a social e psicológica, contudo, 
manter a autoestima durante este período é fundamental. Além dos efeitos colaterais da queda dos cabelos que é consequência direta da quimioterapia, tem-se também efeitos como descamação e queda de unhas e ressecamento da pele, ganho de peso, inchaço, boca seca, alteração de paladar e feridas na boca, tudo colaborando para impactar de forma negativa no humor da pessoa (MORELLE, 2018).

Em poucos países no mundo a beleza é parte tão importante de sua cultura quanto no Brasil, a idolatria a um padrão estético, nem sempre ao alcance de todos, exerce uma verdadeira pressão social acarretando impactos a toda a população e no que tange a um paciente de câncer, que encara nítidos efeitos colaterais essa questão assume um papel ainda mais delicado (INCA, 2013). Pois a preocupação com a estética é apontada por médicos e psicólogos como uma necessidade a ser trabalhada durante o tratamento, uma vez que muitos dos efeitos colaterais podem ser amenizados, fortalecendo a autoestima e trazendo claros benefícios ao tratamento através de atitudes relativamente simples (INCA, 2013).

Por meio de cosméticos especialmente desenvolvidos para pacientes oncológicos alguns destes impactos podem ser minimizados. Os cosméticos oncológicos entregam necessidades como limpeza, hidratação, proteção solar da pele, atrelado a um conceito de cuidado e preservação da pele, sem efeitos nocivos ou, que não tenham interação medicamentosa com os tratamentos indicados pelos médicos oncologistas. Para manter peles e lábios bem hidratados, faz-se o uso de matérias-primas suaves, livres de álcool e ricas em ácidos graxos essenciais, encontrados nos óleos vegetais e nas manteigas naturais, conferindo umectação natural, emoliência e oclusão; os produtos para limpeza da pele não devem conter grande poder de detergência, os tensoativos devem ser suaves e com princípios ativos que auxiliem na regeneração e na hidratação desta pele. Nos cabelos e couro cabeludo os shampoos, condicionadores e máscaras capilares têm de conferir uma limpeza suave, seguidas de emoliência e suavidade nos fios; ademais corantes e fragrâncias devem ser evitados (FRANÇA, 2020).

Nelson Hamerschlak, coordenador do Programa de Oncologia e Hematologia e Transplante de Medula Óssea do Hospital Israelita Albert Einstein, diz que quando o paciente está satisfeito com sua aparência há um impacto direto na tolerância e, possivelmente, até no resultado do tratamento (INCA, 2013). Através da maquiagem é possível alcançar uma fácil harmonização e satisfação com a imagem, já como forma de tratamento terapêutico para indivíduos com algum tipo de insatisfação estética, esta desempenha um papel de camuflagem cosmética ajudando a aliviar os efeitos colaterais 
causados no tratamento de doenças, tendo o poder de modificar até o estado de espírito da pessoa, dando-Ihe maior segurança (FREITAS, 2018).

Os cosméticos são grandes auxiliadores quando relacionados ao bem-estar de pessoas submetidas a tratamento de diversas doenças. Para muitos pacientes, os efeitos colaterais relacionados à aparência são muito preocupantes, muitas vezes afetando profundamente o bem-estar físico, mental e social e desencadeando mudanças negativas na imagem corporal, autoconceito e autoestima. O programa LGFB ajuda mulheres que vivem com câncer a controlar os efeitos colaterais relacionados à aparência (TAGGART et al., 2009).

No Brasil temos o "De bem com Você - A Beleza Contra o Câncer" (DBCV), que surgiu em 2012 e é realizado pela ABIHPEC (Associação Brasileira da Industria de Higiene Pessoal, Perfumaria e Cosméticos) sendo a única que possui permissão para aplicar o programa internacional LGFB, partindo do mesmo princípio de diminuir os efeitos colaterais causados pelo tratamento do câncer através da boa aparência e elevação da autoestima (CARNAÚBA, 2020).

O Instituto ABIHPEC é uma associação civil, autônoma, sem fins lucrativos e surgiu de uma iniciativa da ABIHPEC para canalizar projetos sociais com a missão engajar e mobilizar o setor de higiene pessoal e beleza, bem como a sociedade brasileira, por meio de iniciativas que resgatem a autoestima dos indivíduos e promovam o bem-estar da mulher e inclusão social de todos através de programas como o "Formare" que visa a educação profissional de jovens em condições adversas e o programa "Education for life" no apoio de instituições que promovem a formação profissional na área da beleza para aqueles que se encontram em vulnerabilidade social, entre outros programas sociais, além do objeto de estudo, o "De Bem com Você - A Beleza Contra o Câncer" anteriormente citado (ABIHPEC, 2020).

O programa "De Bem com Você" conta com mais de 30 Instituições de Saúde espalhadas por oito estados brasileiros atendendo no ano passado cerca oito mil pacientes. Para Viggian (2017), presidente do Instituto ABIHPEC, a maquiagem vai muito além de deixar as mulheres mais bonitas, mas sim tem o poder transformador de proporcionar bemestar, sorriso, autoestima e, como disse uma das pacientes após participar da oficina, de torná-las mais confiantes.

Outro exemplo que se pode citar do uso de cosméticos como recurso terapêutico é de uma clínica oncológica no Rio de Janeiro, pois de acordo com a responsável pelo serviço de psicologia, diagnósticos de doenças como o câncer exigem muito do paciente com relação a recursos psíquicos, comprometendo a lidar com a realidade. Neste processo, é 
de extrema importância resgatar o autocuidado, incluindo o uso de cosméticos no cotidiano deste paciente, com a intenção de aumentar a autoestima e encorajar a enfrentar este momento delicado (ESTADÃO, 2017).

Quadros depressivos também estão conexos com maior sensação de dor e desconforto físico. Mesmo para pacientes fora de capacidades terapêuticas, a valorização da autoestima por meio de cuidados estéticos melhora a qualidade de vida (INCA, 2013). Vários esforços têm sido realizados por programas modelos como o LGFB nos Estados Unidos e o seu semelhante De Bem com Você - A Beleza Contra o Câncer no Brasil no que se refere ao suporte psicológico de autoestima às mulheres que estão em tratamento oncológico, entretanto, ainda temos uma notória deficiência nos estudos quanto ao reconhecimento da importância do cosmético como recurso terapêutico.

Para melhor entender a forma com que os cosméticos são relacionados à saúde mental surge a problemática de como estes são subdimensionados e vistos sem atrativos pela comunidade cientifica no que tange a um recurso viável de terapia e promoção da autoestima.

\section{MATERIAL E MÉTODOS}

A metodologia abordada para realização da pesquisa deste trabalho teve dois direcionamentos como pilares. O primeiro caracterizou-se pela revisão de literatura das principais fontes/obras/referências que tratam do tema da pesquisa através do levantamento bibliográfico de fontes científicas (artigos, teses, dissertações) e fontes de divulgação de ideias (revistas e sites) aprofundada em estudo do tipo estado da arte que permiti estabelecer relações com produções anteriores, identificando temáticas recorrentes, apontando novas perspectivas, consolidando uma área de conhecimento (VOSGERAU; ROMANOWSKI, 2014), sendo norteada por publicações tanto de literaturas nacionais quanto internacionais nas bases eletrônicas de dados (Google Scholar, Google e SciELO).

O segundo método utilizado se deu por meio de uma entrevista virtual, com a duração de uma hora via plataforma Zoom, concedida pelo presidente do Instituto ABIHPEC, tendo como foco da entrevista o programa De Bem com Você - A Beleza Contra o Câncer e os principais assuntos tratados foram: a percepção dele sobre como os cosméticos auxiliam na autoestima de pacientes com câncer; a abrangência do programa no território nacional; que tipo de adequações e mudanças foram necessárias realizar para que o programa continuasse o atendimento frente ao cenário da COVID-19 e quais são os programas de expansão deste. 
A coleta das informações do tema se deu através das seguintes etapas:

a) Pesquisa através das palavras-chaves nos buscadores de informações;

b) Levantamento de dados de 27 artigos além de outros tipos de documentos eletrônicos de sites;

c) Leitura parcial dos conteúdos levantados para seleção do material a ser utilizado;

d) Leitura na íntegra dos conteúdos selecionados.

e) Entrevista concedida pelo presidente do Instituto ABIHPEC, Cláudio Viggian, sobre o programa De Bem com Você - A Beleza Contra o Câncer.

A procura dos textos foi elaborada através do uso do operador de busca booleano, combinando dois ou mais termos, a fim de refinar a pesquisa tornando-a mais detalhada e restrita. Para tal, as palavras AND, OR e NOT ("E, OU e NÃO) são utilizadas, entretanto, para realização desta, a palavra AND (E) foi usada a fim de promover combinações resultando assim em uma busca mais precisa (SAKS, 2005). As palavras-chaves combinadas foram look good feel better AND self-esteem ( 862 mil resultados); Instituto Abihpec AND câncer ( 119 mil resultados); autoestima AND câncer ( 25,3 mil resultados); autoestima AND tratamento oncológico ( 9,87 mil resultados); maquiagem AND autoestima ( 6,28mil resultados); câncer AND cosméticos AND autoestima ( 2,5 mil resultados), conforme figura 1.

$\mathrm{Na}$ etapa da entrevista concedida pelo presidente do Instituto ABIHPEC, Cláudio Viggian, este relatou que as oficinas de automaquiagem, ministradas por maquiadoras profissionais voluntárias, geralmente são realizadas nas instituições em que as pacientes estão em tratamento e que este é um momento de acolhimento e transformação pois além de ensinar técnicas de maquiagem de uma forma simples, como redesenhar as sobrancelhas, ruborizar as maçãs do rosto, colorir os lábios, dar aulas de amarração de lenços e dicas de cuidados com a pele, também enfatiza que esta fase pela qual as pacientes estão passando é passageira e que o programa tem a missão de ampará-las emocionalmente, tudo no sentido de melhorar a aparência que foi prejudicada pelo tratamento oncológico, onde muitas vezes entram mulheres retraídas e deprimidas e saem mulheres com um animo muito melhor.

Após conclusão das etapas acima citadas, foram selecionados 19 artigos, também relatórios de pesquisa, reportagens, depoimentos além da entrevista acima explanada. Esta baixa demanda de artigos elegidos se deve a uma lacuna expressiva existente entre o tema cosmético dentro de uma perspectiva terapêutica de elevação da autoestima, pois apesar da grande quantidade de resultados mostrados após as buscas, em sua maioria os conteúdos 
abordados eram específicos da questão medicinal da doença.

Os critérios de escolha foram de acordo com a relevância do conteúdo sobre a influência que os cosméticos exercem no quesito de elevar a autoestima do indivíduo, comprovado por meio de dados estáticos de relatórios de pesquisa, pontos de vista de profissionais da área da saúde, programas sociais que visam promover a autoestima das mulheres durante o tratamento da doença, vídeo aulas de automaquiagem, reportagens, entrevista e relatos de pacientes.

Figura 1 - Processo de seleção dos artigos

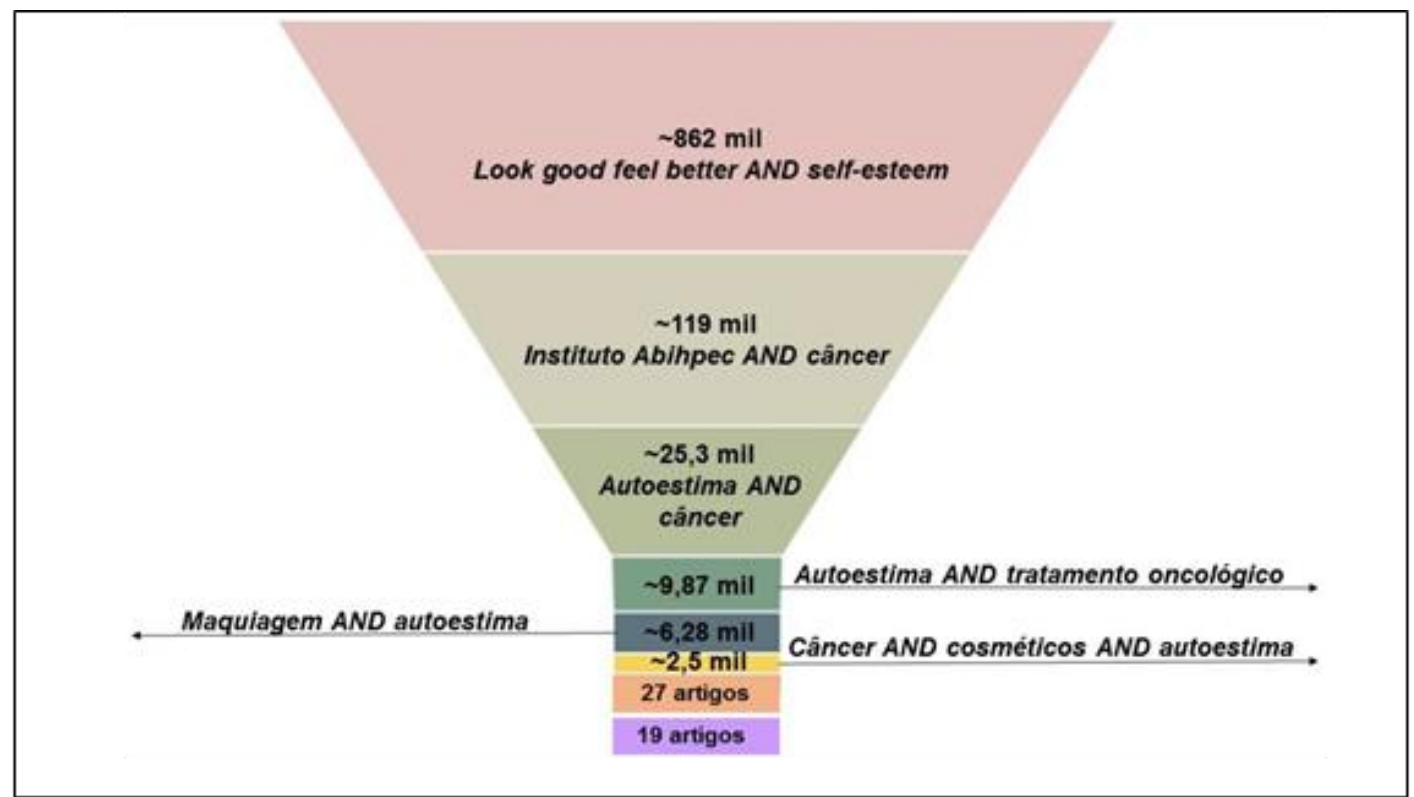

Fonte: Autores (2020)

\section{RESULTADOS E DISCUSSÃO}

Em casos em que se tem uma doença como agravante, os cosméticos podem ser uma fonte de bem-estar e confiança, podendo este fortalecimento emocional ajudar na recuperação do paciente, pois a elevação da autoestima estimula as defesas naturais do organismo (ESTADÃO, 2017).

Roark (2008) cita que em duas décadas de programa desde seu lançamento em 1989, o LGFB já havia transformado a vida de quase 600.000 mulheres de todas as idades e etnias e com todas as formas de câncer, mitigando as mudanças que os tratamentos causam na aparência afetando a qualidade de vida, confiança, e capacidade de lidar com a doença. Um estudo em grande escala realizado no Laboratório de Neurociência Afetiva 
nos EUA investigou as relações entre as medidas fisiológicas do estilo afetivo, bem-estar psicológico e função imunológica, o qual apontou como resultado que os indivíduos caracterizados por um estilo afetivo mais negativo mostraram uma resposta imunológica mais fraca apresentando maior risco de adoecer do que aqueles com um estilo afetivo mais positivo (BARAK, 2006).

No ano de 2002, o LGBF patrocionou uma pesquisa conduzida pela Harris Interactive, uma empresa de pesquisa de mercado e consultoria global, com 267 mulheres tratadas de câncer nos últimos 5 anos para compreender melhor a qualidade de vida geral durante o tratamento e a relação entre a aparência de uma mulher e seus sentidos de bemestar (ROARK, 2008). O gráfico da figura 2 mostra alguns destes resultados.

Figura 2 - Qualidade de vida durante tratamento oncológico

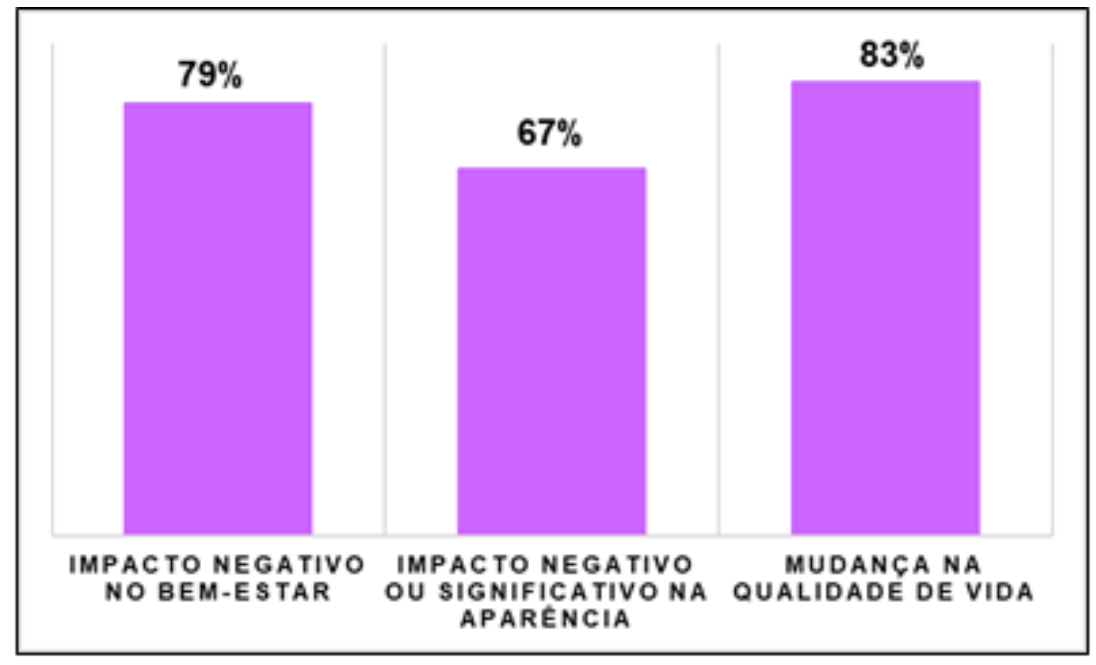

Fonte: Autores (2020)

Barak (2006) diz que os sentidos de bem-estar podem estar relacionados às respostas emocionais, pois estas fazem parte de interações fisiológicas complexas comprometendo a capacidade do corpo de ser sadio ou até mesmo de resistir ou preponderar doenças. Assim sendo, o estado emocional influência na causa e evolução da doença e também auxilia para a fragilidade ou resistência.

Outra pesquisa feita durante o mês de março de 2012 de forma online nos Estados Unidos pela Harris Interactive em nome do programa LGFB, destaca como os efeitos colaterais do tratamento do câncer, embora de natureza cosmética, afetam a autoestima e a confiança, bem como os relacionamentos em casa e no local de trabalho. O estudo foi realizado com 1226 mulheres acima de 25 anos de idade e relata sobre a importância que 
é para a mulher ter uma boa aparência, 95\% das mulheres que não tiveram câncer e 97\% das mulheres com câncer relataram que há uma conexão entre sua aparência e como se sentem (SURVEY, 2012). Alguns dos resultados desta pesquisa podem ser visto nas figuras 3 , 4 e 5 .

Figura 3 - Aspectos físicos com maior impacto na autoestima antes de tratar o câncer.

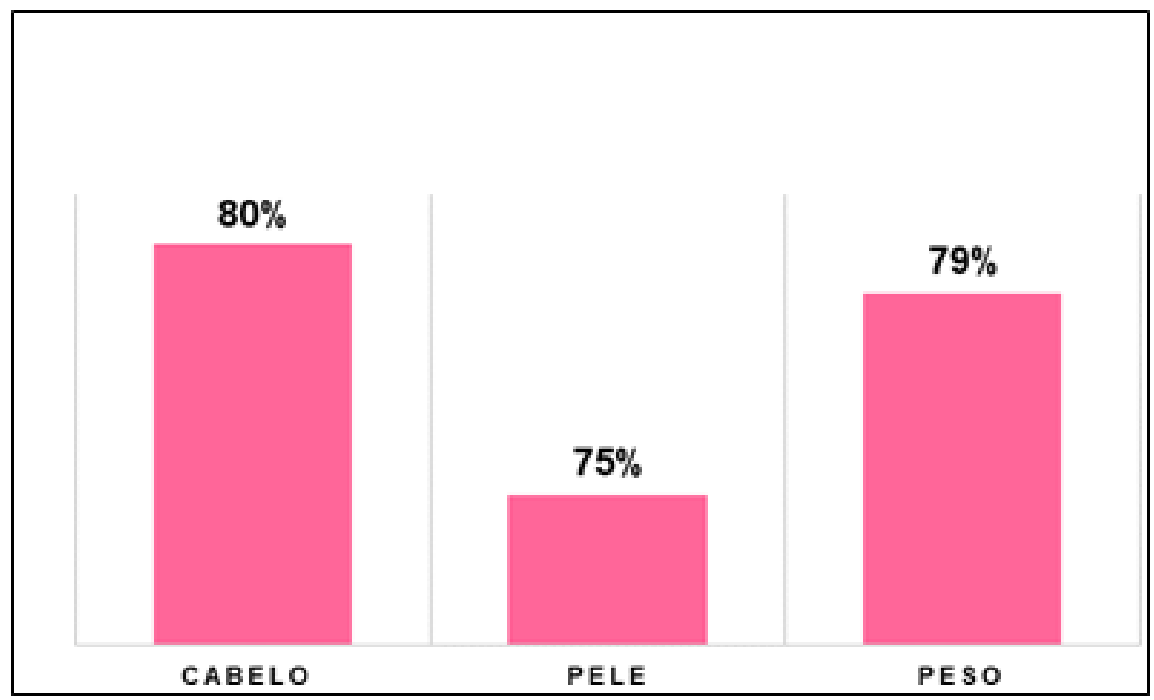

Fonte: Autores (2020)

Duas em cada cinco pacientes tiveram queda de cabelo no período de tratar a doença; $57 \%$ perderam todo o cabelo, $14 \%$ rasparam a cabeça e $25 \%$ usaram peruca durante o tratamento após a perda dos cabelos; $67 \%$ experimentaram mudanças no peso e $51 \%$ das mulheres enfermas notaram mudanças em sua pele como resultado do tratamento contra o câncer (SURVEY, 2012).

A Dra. Mary-Jane Massie, psiquiatra especializada no tratamento psicológico de pessoas com câncer e suas famílias diz que há uma cascata de emoções após um diagnóstico e tratamento de câncer como perder o cabelo, mudanças de peso e aparência, todos os sinais externos de câncer roubam o senso de identidade e a autoconfiança (WIRE, 2012).

Dados da pesquisa apontam que o tratamento do câncer tem um impacto negativo na confiança e no controle que as mulheres têm sobre sua aparência conforme figura 4. 
Figura 4 - Impactos negativos do tratamento do câncer

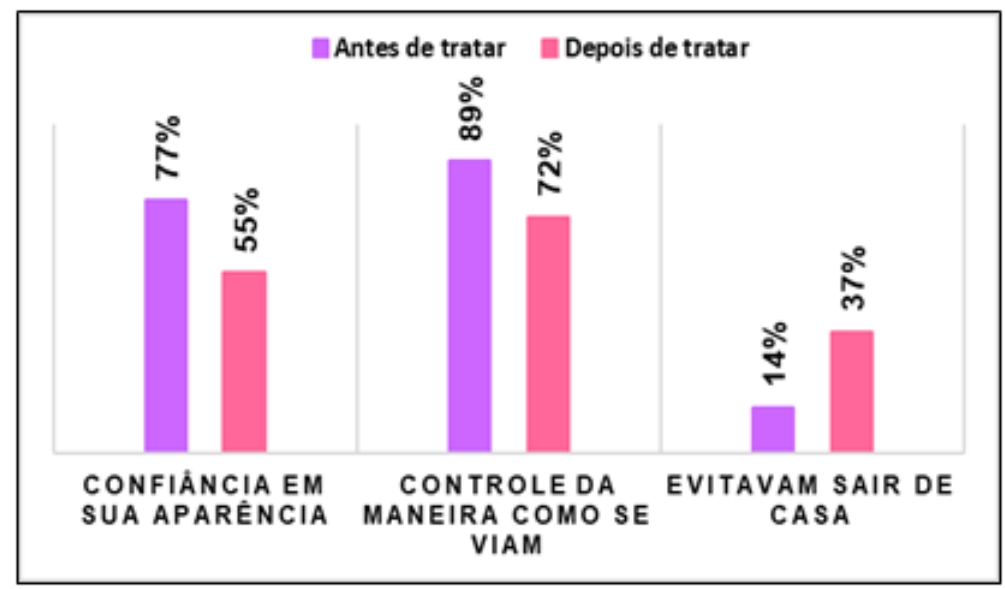

Fonte: Autores (2020)

Os efeitos colaterais do câncer relacionados à aparência também afetam a maneira como as mulheres são tratadas pelas pessoas do seu entorno, $33 \%$ das respondentes da pesquisa disseram que aparência alterada tinha um impacto negativo em seu relacionamento com o cônjuge; $25 \%$ tiveram dificuldades de relacionamento com os filhos e $24 \%$ com amigos; $63 \%$ disseram que as pessoas não sabiam como agir quando as viam e apenas 27\% não notaram diferença em relação ao convívio com colegas de trabalho (SURVEY, 2012).

Por outro lado, ao abordar os efeitos colaterais relacionados a aparência que possam surgir durante as terapias do câncer pode ser benéfico para a autoestima da paciente como mostrado na figura 5 .

Figura 5 - Benefícios de participar do programa LGBF

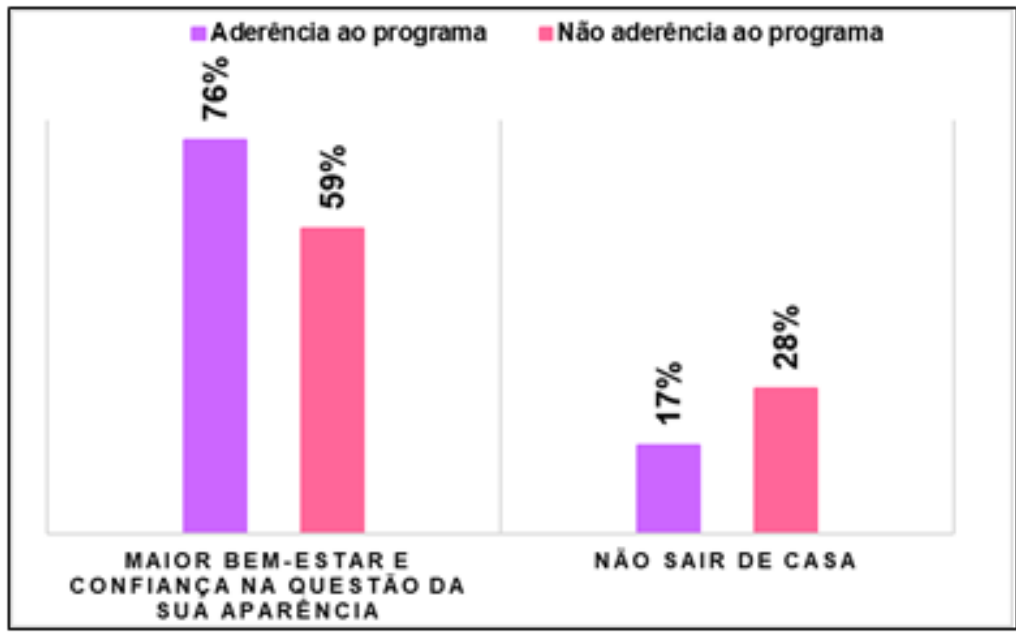

Fonte: Autores (2020) 
Além do que $57 \%$ das mulheres que abordaram o assunto falaram que lidar com a situação resultou positivamente na maneira de como as pessoas as tratavam, 55\% disseram ter um impacto positivo nas "experiências sociais" e $60 \%$ se sentiram no controle da situação após abordagem do assunto. O programa mostra seus benefícios através das participantes deste que se sentiram mais confiantes e no controle da situação do que aquelas que lidaram com seus efeitos colaterais sem usar o programa (SURVEY, 2012) . Louanne Roark, diretora executiva da Fundação Look Good Feel Better do Personal Care Products Council diz que viver com câncer pode ser opressor e o objetivo do programa é ajudar essas pessoas a se sentirem mais confiantes para se exporem, seja para trabalhar, levar os filhos à escola, fazer compras ou apenas se olhar no espelho em casa (MONINGER, 2017).

A editora de beleza Caitlin Kiernan e autora do Pretty Sick: The Beauty Guide for Women With Cancer, livro que escreveu depois de se submeter a um tratamento para câncer de mama, fala que além da preocupação com a saúde também queria ter uma aparência normal, especialmente porque estava trabalhando na indústria da beleza e que uma das entrevistadas de seu livro relatou o quão importante que era ter uma aparência saudável para 0 bem de seus filhos pequenos. Kiernan ainda afirma que independentemente do motivo os tratamentos de beleza são uma terapia adjuvante aos tratamentos oncológicos (MONINGER, 2017).

Dados coletados da terceira pesquisa global anual (2018/2019) dos participantes do programa LGFB com mais de 26 mil pessoas atendidas em dezesseis países, do qual o Brasil fez parte estando presente por meio da parceria com o De Bem com Você - a Beleza Contra o Câncer (DBCV), nos mostra resultados de profundo impacto positivo às mulheres no tratamento do câncer, independentemente do país ou cultura (WIRE, 2020). A figura 6 mostra os resultados percentuais da pesquisa a nível mundial e a nível Brasil no qual o programa De Bem Com Você - A Beleza Contra o Câncer colaborou com a participação de 5.325 pessoas em 23 cidades (ABIHPEC, 2020). 
Figura 6 - Terceira pesquisa global anual do programa LGFB

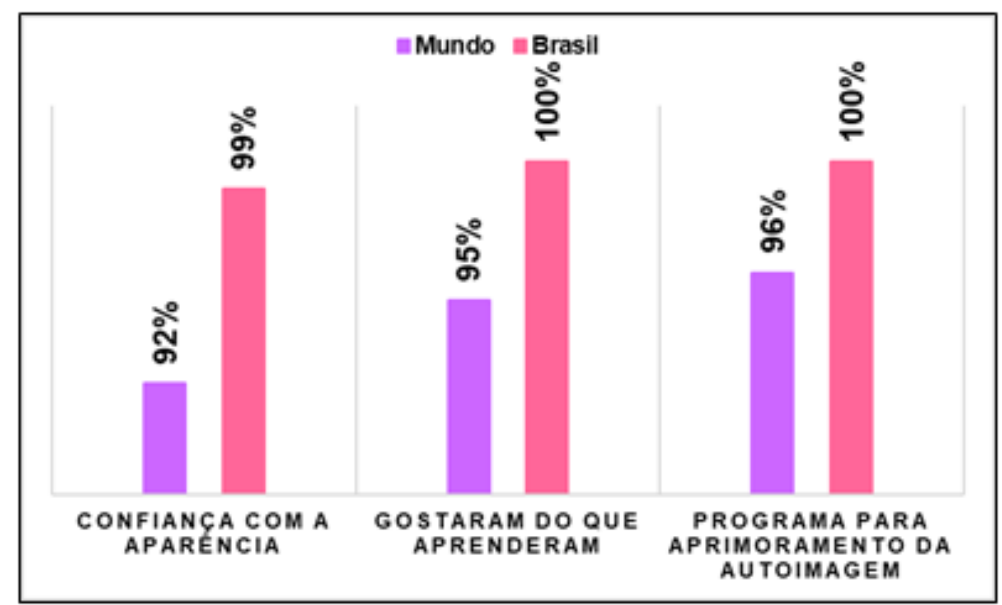

Fonte: Autores (2020)

Desde 1989 o programa empoderou 2 milhões de mulheres em 27 países ao ajudálas a recuperar o senso de controle, confiança e autoestima, fatores que são primordiais ao bem-estar (WIRE, 2020).

Visto tamanha importância e os benefícios que traz na melhora da autoestima de mulheres em tratamento de câncer, o projeto De Bem com Você - A Beleza Contra o Câncer tem como premissa priorizar quem mais precisa, sendo que mais de $90 \%$ das pacientes fazem tratamento da doença pelo Sistema Único de Saúde (SUS), conforme declara Claudio Viggiani, presidente do Instituto (ESTADÃO, 2017).

Durante a entrevista com o presidente do Instituto ABIHPEC, no Brasil, desde a sua fundação no final de 2012, o Programa de Bem com Você - A Beleza Contra o Câncer já atendeu mais de 30 mil mulheres em tratamento oncológico até os dias de hoje, sendo que só no ano passado foram mais de 8 mil mulheres atendidas e que existe um programa de expansão onde a meta é conseguir atender 20 mil mulheres por ano. Entretanto, neste ano os números foram menos expressivos por conta da pandemia da Covid-19 onde mudanças e adequações visando a segurança de todos foram necessárias e as oficinas passaram a ser em plataformas virtuais além também da inserção de Lives relacionadas ao tema, e mesmo quando o retorno das oficinas presenciais voltarem a ser uma realidade, estas não deixarão de fazer parte do programa já que são vistas como uma possibilidade extra de atendimento alcançando as pacientes que de alguma forma não podem estar presente nas reuniões.

No programa LGFB a nível mundial, os Estados Unidos ainda é a referência atendendo cerca de 60 mil pacientes por ano, todavia, podemos dizer que o Brasil está em 
ascensão sendo destaque nos últimos três anos como o programa que teve maior crescimento dentro da rede LGFB.

Entre 2016 e 2017, o projeto Avaliação do Bem-Estar de Pacientes que Fazem Uso de Tratamento Oncológico recrutou 80 voluntárias com câncer de mama a fim de mensurar a melhora do estado psicológico após workshops de beleza. Os resultados publicados após a conclusão do projeto, mostraram que, entre as participantes, houve um decréscimo da tristeza após as intervenções (CARNAÚBA, 2020). Ato que pode ser corroborado pela psicóloga Mariana Cavalcante da Associação Brasileira de Linfoma e Leucemia (Abrale), quando destaca que o autocuidado é uma forma de se resgatar, pois proporciona uma maior leveza ao paciente, impactando de maneira positiva em seu tratamento (FRANQUILINO, 2018).

Um outro estudo realizado por pesquisadores da Unifesp com o apoio do Instituto Paulista de Cancerologia (IPC), teve como propósito aferir o efeito das intervenções de beleza sobre as voluntárias através dos métodos da escala de autoestima de Rosenberg (EAR), baseado na pontuação obtida em um questionário composto por dez questões fechadas e a Facial Action Coding System (Facs) que capta e codifica os movimentos de músculos faciais individuais a partir de mudanças momentâneas distintas na aparência facial. Apenas as respostas ao questionário foram consideradas insuficientes para avaliar com precisão as alterações no nível de bem-estar das voluntárias. Entretanto por meio do software instalado em um tablet e acoplado a um espelho, foi possível detectar as expressões faciais enquanto se maquilavam e as observações foram divididas em etapas registrando-se os aspectos fisionômicos a cada milissegundo. O uso do batom inspirou mais confiança enquanto ao colocar o lenço na cabeça gerou expressões de raiva e aversão - o que pode estar associado a perda de cabelos devido à quimioterapia. $O$ resultado dos testes não revelou pessoas mais positivas, mas menos negativas (CARNAÚBA, 2020).

Os construtos utilizados para embasamento desta pesquisa encontram-se no Quadro 1. 


\section{Quadro 1 - Artigos selecionados para embasamento do trabalho}

\begin{tabular}{|c|c|c|c|c|}
\hline ID & Artigo & Autores & Ano & Principais achados \\
\hline 1 & $\begin{array}{c}\text { The impact of the use of cosmetics } \\
\text { on the well-being of patients } \\
\text { undergoing cancer treatment }\end{array}$ & $\begin{array}{l}\text { Luana Alexandrino Rodrigues } \\
\text { da Silva }\end{array}$ & 2018 & $\begin{array}{l}\text { Em virtude das mudanças sofridas pelo corpo } \\
\text { devido ao tratamento de câncer, alterações } \\
\text { negativas na autoestima são inevitáveis. } \\
\text { Estudo avalia as emoções através de software } \\
\text { que codifica as reaçôes faciais involuntárias } \\
\text { promovidos pelo uso da maquiagem. }\end{array}$ \\
\hline 2 & $\begin{array}{c}\text { Use of cosmetic makeup, to } \\
\text { camouflage lesions on the face, } \\
\text { caused by:melasma; rosacea and } \\
\text { acne }\end{array}$ & $\begin{array}{l}\text { Norma Nelson Gonçalves } \\
\text { Freitas; Fabiana Durante de } \\
\text { Medeiros }\end{array}$ & 2018 & $\begin{array}{l}\text { O desempenho da maquiagem como } \\
\text { camuflagem cosmética no auxílio da } \\
\text { reconstrução da imagem e a da } \\
\text { autoconfiança abaladas pelos efeitos } \\
\text { colaterais de tratamento de doenças. }\end{array}$ \\
\hline 3 & $\begin{array}{l}\text { More Than Skin Deep: Improving } \\
\text { Physical Appearance After Cancer }\end{array}$ & Jeannette Moninger & 2017 & $\begin{array}{c}\text { Relatos de pacientes com câncer sobre como } \\
\text { este afetou suas vidas; quais são as } \\
\text { impressões das mulheres que estão em } \\
\text { tratamento e participaram do LGFB tem sobre } \\
\text { o programa. }\end{array}$ \\
\hline 4 & Cosmetics Action and Reaction & \begin{tabular}{|c|} 
Dryelle Feitosa Mota; Likaele \\
Moreira Nunes; Luiza Brenna \\
Soares de Souza; Luiza \\
Tamires da Silva Oliveira; \\
Amanda Karine de Sousa
\end{tabular} & 2014 & $\begin{array}{l}\text { A utilização de cosméticos e suas mais } \\
\text { diversas aplicações e cuidados no uso destes } \\
\text { produtos para não causar danos a pele. }\end{array}$ \\
\hline 5 & $\begin{array}{l}\text { Vanity Economics: An Economic } \\
\text { Exploration of Sex, Marriage and } \\
\text { Family }\end{array}$ & Chengze Simon Fan & 2014 & $\begin{array}{c}\text { Relato sobre uma pesquisa inicial a respeito da } \\
\text { economia familiar e comportamento social, } \\
\text { mas que posteriormente aborda a maneira com } \\
\text { que os pais passam seus hábitos sobre } \\
\text { riqueza a seus filhos para que perpetuem o } \\
\text { altopadrão social, estabelecendo assim uma } \\
\text { relação entre este status social com a } \\
\text { autoestima do indivíduo. }\end{array}$ \\
\hline 6 & $\begin{array}{l}\text { hderstand women's vanity using } \\
\text { self-steem and personality }\end{array}$ & $\begin{array}{l}\text { Cátia Fabíola Parreira de } \\
\text { Avelar; Ricardo Teixeira } \\
\text { Veiga }\end{array}$ & 2013 & $\begin{array}{l}\text { A relação da autoestima e variáveis de } \\
\text { personalidade na vaidade feminina e como } \\
\text { podem influenciar no âmbito estético, não só } \\
\text { para mulheres que enfrentam um tratamento } \\
\text { de câncer, mas de forma geral. }\end{array}$ \\
\hline 7 & $\begin{array}{l}\text { It's no secret, looking good is } \\
\text { important to women, regardless } \\
\text { of whether or not they have or } \\
\text { have had cancer. }\end{array}$ & Harris Survey & 2012 & $\begin{array}{c}\text { Estudo que salienta a importância que as } \\
\text { mulheres dão a aparência, principalmente } \\
\text { para as que enfrentam o tratamento do } \\
\text { câncer, pois afeta a imagem com que elas se } \\
\text { enxergam e também na forma com que os } \\
\text { outros Ihetratam, sendo primordial } \\
\text { restabelecer esta autoestima e confiança } \\
\text { em si. } \\
\end{array}$ \\
\hline 8 & $\begin{array}{l}\text { A reflection on the influence of } \\
\text { aesthetics on self-esteem, self- } \\
\text { motivation and human well-being }\end{array}$ & Tamila J. Borba & 2011 & $\begin{array}{l}\text { A relação dos tratamentos de beleza com a } \\
\text { autoestima, como esta influência no } \\
\text { psicológico do indivíduo e através de quais } \\
\text { atitudes pode ser fortalecida para se ter uma } \\
\text { vida de qualidade. }\end{array}$ \\
\hline 9 & $\begin{array}{l}\text { Mirror, mirror of mine, is there } \\
\text { anyone more vain than me? } \\
\text { Female vanity and its influence } \\
\text { on self-esteem and consumption } \\
\text { of aesthetic procedures }\end{array}$ & $\begin{array}{l}\text { Vivian lara Strehlau; Danny } \\
\text { Pimentel Claro; Silvio } \\
\text { Abrahão Laban Neto }\end{array}$ & 2010 & $\begin{array}{l}\text { A relação entre a vaidade feminina com a } \\
\text { autoestima e procedimentos estéticos, pois } \\
\text { quanto maior for o grau de vaidade da mulher } \\
\text { maior influência há de sua autoestima com } \\
\text { sua aparência, uma vez que esta vaidade } \\
\text { caracteriza uma preocupação e uma visão } \\
\text { positiva da aparência física e realizações. }\end{array}$ \\
\hline 10 & $\begin{array}{l}\text { Self-esteem and Self-image: The } \\
\text { relationship with aesthetics }\end{array}$ & $\begin{array}{l}\text { Flavia Monique Floriani; } \\
\text { Márgara Dayana da Silva } \\
\text { Marcante }\end{array}$ & 2010 & $\begin{array}{c}\text { O conceito de autoestima como auto aceitação } \\
\text { ou auto rejeição da própria imagem, sua } \\
\text { influência na saúde mental e a importância da } \\
\text { estética neste assunto. }\end{array}$ \\
\hline 11 & $\begin{array}{c}\text { Look good feel better workshops: A } \\
\text { "big lift" for women with cancer }\end{array}$ & \begin{tabular}{|c|} 
Linda R. Taggart; Laura \\
Ozolins; Joyce Nyhof Young
\end{tabular} & 2009 & $\begin{array}{c}\text { O impacto dos workshops LGFB na autoimagem, } \\
\text { interações sociais, apoio social percebido e } \\
\text { ansiedade. }\end{array}$ \\
\hline
\end{tabular}




\begin{tabular}{|c|c|c|c|c|}
\hline 12 & $\begin{array}{l}\text { 'Normalizing' female cancer } \\
\text { patients: Look good, feel better } \\
\text { and other image programs }\end{array}$ & Karen Kendrick & 2008 & $\begin{array}{l}\text { A recuperação da feminilidade e da aparência } \\
\text { feminina pelo incentivo do uso de cosméticos } \\
\text { para aumentar sua autoestima deixando-a } \\
\text { mais autoconfiante durante o processo de } \\
\text { tratamentos oncológicos. }\end{array}$ \\
\hline 13 & $\begin{array}{l}\text { Look good...feel better helps } \\
\text { people battling cancer help } \\
\text { themselves }\end{array}$ & Louanne Roark & 2008 & $\begin{array}{c}\text { Benefícios que o programa LGFB traz para } \\
\text { as participantes deste além de dados de uma } \\
\text { pesquisa realizada pelo LGFB para } \\
\text { compreender melhor a qualidade de vida } \\
\text { geral durante o tratamento e a relação entre } \\
\text { a aparência de uma mulher e seus sentidos } \\
\text { de bem-estar. }\end{array}$ \\
\hline 14 & $\begin{array}{l}\text { Adolescents of different social } \\
\text { classes giving value of beauty } \\
\text { and intelligence }\end{array}$ & $\begin{array}{l}\text { Gizelle Regina Gomes; } \\
\text { Sandro Caramaschi }\end{array}$ & 2007 & $\begin{array}{l}\text { Estudo realizado com adolescentes de } \\
\text { gêneros e classes socioeconômicas distintas } \\
\text { refletindo sobre o valor que atribuem a beleza } \\
\text { e a inteligência. Relativo a beleza, os } \\
\text { indivíduos mais vaidosos tendem a ser mais } \\
\text { satisfeitos e tolerantes consigo mesmos, ao } \\
\text { contrário dos menos vaidosos que acabam se } \\
\text { fechando e criando uma espécie de armadilha. }\end{array}$ \\
\hline 15 & $\begin{array}{l}\text { The immune system and } \\
\text { happiness }\end{array}$ & Yoram Barak & 2006 & $\begin{array}{l}\text { Estudo que compreende relações entre as } \\
\text { medidas fisiológicas do estilo afetivo, bem- } \\
\text { estar psicológico e função imunológica e como } \\
\text { o estado emocional do indivíduo influencia de } \\
\text { forma positiva ou negativa na causa e } \\
\text { evolução da doença. }\end{array}$ \\
\hline 16 & $\begin{array}{l}\text { Hedonic consequences } \\
\text { of social comparison: A } \\
\text { contrast of happy and } \\
\text { unhappy people }\end{array}$ & $\begin{array}{c}\text { Sonja Lyubomirsky; Lee } \\
\text { Ross }\end{array}$ & 1997 & $\begin{array}{c}\text { Baseado em dois estudos cujo intuito foi de } \\
\text { testar hipóteses sobre a resposta sensível de } \\
\text { indivíduos avaliados como felizes e infelizes, } \\
\text { estabelecendo uma associação com a vaidade } \\
\text { e autoestima, uma vez que indivíduos vadosos } \\
\text { tendem a se enquadrar em um modelo de } \\
\text { autovalorização. }\end{array}$ \\
\hline 17 & $\begin{array}{c}\text { Trait Aspects of Vanity: } \\
\text { Measurement and Relevance to } \\
\text { Consumer Behavior }\end{array}$ & $\begin{array}{l}\text { Richard G. Netemeyer, Scot } \\
\text { Burton, Donald R. } \\
\text { Lichtenstein }\end{array}$ & 1995 & $\begin{array}{l}\text { Estudo que aponta vaidade a construtos e a } \\
\text { comportamentos, estabelecendo dois pilares } \\
\text { ao conceito de vaidade, um relativo à visão } \\
\text { positiva e até mesmo exagerada da questão } \\
\text { física e a outra das realizações pessoais. }\end{array}$ \\
\hline
\end{tabular}

Fonte: Autores (2020)

\section{CONSIDERAÇÕES FINAIS}

Resgatando o propósito supracitado na introdução, além de buscar entender a influência que os cosméticos possuem do ponto de vista terapêutico como promoção de autoestima para pacientes em tratamento de câncer, por meio de programas sociais, relatos, estudos e reportagens, podemos dizer que em termos de valência emocional este atua como uma ferramenta de apoio durante o tratamento, dado que não muda apenas a aparência física da pessoa, mas também a maneira como ela se vê de uma forma positiva.

Uma vez relacionado a saúde na intenção de proporcionar uma melhor qualidade de vida, os cosméticos não podem ser conceituados e tratados como futilidades somente relativo à estética. $\mathrm{O}$ impacto da maquiagem em mulheres, principalmente nas que se encontram em tratamento oncológico, não se limita a deixá- las mais bonitas, o efeito de transformação proporcionado por esta vai além, se reverte em autoestima, auxiliando também 
no cuidado de corações angustiados.

Todavia cabe ressaltar que o cosmético não é um recurso singular para o resgate da autoestima, outras ferramentas como terapias diversas, preferências individuais, atividades físicas, evitar comparações com outrem, se cercar de pessoas que lhe fazem bem são formas de aprofundar o autoconhecimento que a mulher tem de si mesma além da autoconfiança neste período de vulnerabilidade física e emocional promovendo um aumento na qualidade de vida durante essa fase de tratamento.

Durante a realização desta pesquisa evidenciou-se uma baixa aderência de estudos relacionados nesta área de conhecimento, já que dificuldades de encontrar referências relevantes se fizeram presentes para a execução deste trabalho, notando- se uma de falta de interesse por parte da comunidade científica que pouco aborda sobre o assunto, averiguando-se assim a necessidade de uma maior extensão dos estudos neste campo da ciência para melhor compreensão e consolidação dos impactos benéficos que os cosméticos oportunizam na autoestima e consequente qualidade de vida durante 0 período de tratamento do câncer.

\section{AGRADECIMENTOS}

Agradecemos primeiramente a Deus por ter nos mantido na trilha certa durante este projeto de pesquisa com saúde e forças para chegar até o final. Somos gratas à nossa família pelo suporte que sempre nos deram durante toda a nossa vida. Também gostaríamos de agradecer ao Sr. Cláudio Viggian, presidente do Instituto ABIHPEC, por toda a sua disponibilidade, recursos e simpatia a nós concedido em entrevista sobre o programa De Bem com Você - A Beleza Contra o Câncer.

\section{REFERÊNCIAS}

ABIHPEC, Instituto. Pesquisa global do de bem com você destaca a importância do programa para a autoestima de mulheres em tratamento contra o câncer. 2020. Disponível em: https://institutoabihpec.org.br/de-bem-com-voce/pesquisa- global-do-de-bem-comvoce-destaca-a-importancia-do-programa/. Acesso em: 27 set. 2020.

AVELAR, Cátia Fabíola Parreira de; VEIGA, Ricardo Teixeira. Como entender a vaidade feminina utilizando a autoestima e a personalidade. Revista de Administração de Empresas, 
[S.L.], v. 53, n. 4, p. 338-349, ago. 2013. FapUNIFESP (SciELO). http://dx.doi.org/10.1590/s0034-75902013000400002

BARAK, Yoram. The immune system and happiness. Autoimmunity Reviews, [S.L.], v. 5, n. 8, p. 523-527, out. 2006. Elsevier BV. http://dx.doi.org/10.1016/j.autrev.2006.02.010.

BETTER, Fundação Look Good Feel. Look Good Feel Better. 2020. Disponível em: https://lookgoodfeelbetter.org/. Acesso em: 25 jul. 2020.

BORBA, Tamila J. Uma Reflexão Sobre A Influência Da Estética Na Auto Estima, AutoMotivação E Bem-Estar Do Ser Humano. 2011. 21 f. TCC (Graduação) - Curso de Cosmetologia e Estética, Universidade do Vale do Itajaí - Univali, Balneário Camboriú, 2011. Disponível em: http://siaibib01.univali.br/pdf/Tamila\%20Josiane\%20Borba.pdf. Acesso em: 24 ago. 2020.

CARNAÚBA, Valquíria. Medindo as emoções: projeto de extensão, executado em parceria com o instituto abihpec, avaliou oscilações emocionais em mulheres acometidas por câncer de mama após aplicação de cosméticos faciais. Projeto de extensão, executado em parceria com o Instituto Abihpec, avaliou oscilações emocionais em mulheres acometidas por câncer de mama após aplicação de cosméticos faciais. 2020. Disponível em: https://www.unifesp.br/edicao-atual- entreteses/item/4261-medindo-as-emocoes. Acesso em: 27 set. 2020

ESTADÃO. O poder da maquiagem na luta contra o câncer. 2017. Disponível em: http://patrocinados.estadao.com.br/abihpec/2017/03/02/o-poder-da-maquiagem-na-lutacontra-o-cancer/. Acesso em: 23 set. 2020.

ESTADÃO: Aliados do dia a dia, cosméticos ajudam a promover autoestima. [S.L.], 11 set. 2017. Disponível em: http://patrocinados.estadao.com.br/abihpec/2017/09/11/aliados-dodia-a-dia-cosmeticos-ajudam-a-promover-autoestima-e-bem-estar/. Acesso em: 20 jul. 2020.

FAN, Chengze Simon. Economia da vaidade: Uma exploração econômica de sexo, casamento e família. Reino Unido: Edward Elgar, 2014. 283 p. 
FLORIANI, Flavia Monique; MARCANTE, Márgara Dayana da Silva. Auto-Estima E AutoImagem: A Relação Com A Estética. 2010. 15 f. TCC (Graduação) - Curso de Cosmetologia e Estética, Universidade do Vale do Itajaí - Univali, Balneário Camboriú, 2010. Disponível em: http://siaibib01.univali.br/pdf/Flavia\%20Monique\%20Floriani,\%20M\%C3\%A1rgara\%2 0Dayana\%20da\%20Silva\%20Marcante.pdf. Acesso em: 25 ago. 2020.

FRANÇA, Paula. COSMÉTICOS ONCOLÓGICOS. Disponível em: https://www.oncofisio.com.br /cosmeticos-oncologicos. Acesso em: 16 dez. 2020.

FRANQUILINO, Erica. Cosméticos para pacientes oncológicos. 2018. Disponível em: https://www.cosmeticsonline.com.br/materia/40. Acesso em: 27 set. 2020.

FREITAS, Norma Nelson Gonçalves. Uso da maquiagem cosmética, para camuflar lesões na face, causadas por: melasma; rosácea e acne. 2018. 37 f. TCC (Graduação) - Curso de E Tecnologia em Cosmetologia e Estética, Universidade do Sul de Santa Catarina -Unisul, Santa Catarina, 2018. Disponível em: https://www.riuni.unisul.br/bitstream/handle/12345/ 6811/TRABALHO\%20DE\%20CONCLUSAO\%20DE\%20CURSO\%202018\%20REUNI.pdf ?sequence=1\&isAllowed=y. Acesso em: 12 nov. 2020

GOMES, Gizelle Regina; CARAMASCHI, Sandro. Valorização de beleza e inteligência por adolescentes de diferentes classes sociais. Psicologia em Estudo, [S.L.], v. 12, n. 2, p. 295303, ago. 2007. FapUNIFESP (SciELO). http://dx.doi.org/10.1590/s141373722007000200010 .

INCA, Instituto Nacional de Câncer José Alencar Gomes da Silva -. Beleza terapêutica. A valorização da autoestima como auxiliar no tratamento do câncer: autoestima é fundamental. Rede Câncer, Rio de Janeiro, v. 2, n. 21, p. 24-27, 21 abr. 2013. Trimestral. Disponível em: https://www.inca.gov.br/publicacoes/revistas/revista- rede-cancer-21. Acesso em: 30 ago. 2020.

LAPORTE, Gustavo Andreazza; LEONARDI, Dilma Francisco. Transtorno de estresse póstraumático em pacientes com sequelas de queimaduras. Rev Bras Queimaduras, Porto Alegre, v. 9, n. 3, p. 105-114, jul. 2010. Disponível em: http://www.rbqueimaduras.com.br/ details/44/pt-BR/transtorno-de-estresse-pos- traumatico-em-pacientes-com-sequelas-de- 
queimaduras. Acesso em: 17 dez. 2020.

LYUBOMIRSKY, Sonja; ROSS, Lee. Hedonic consequences of social comparison: a contrast of happy and unhappy people. Journal Of Personality And Social Psychology, [S.L.], v. 73, n. 6, p. 1141-1157, 1997. American Psychological Association (APA). http://dx.doi.org/10.1037/0022-3514.73.6.1141.

KENDRICK, Karen. 'Normalizing' female cancer patients: look good, feel betterand other image programs. Disability \& Society, [S.L.], v. 23, n. 3, p. 259-269, 23 abr. 2008. Informa UK Limited. http://dx.doi.org/10.1080/09687590801954042.

MONINGER, Jeannette. Mais do que superficialmente: melhorando a aparência física após o câncer. Cure, [S.I], v. 1, n. 1, p. 15-17, 25 ago. 2017. Disponível em: https://www.curetoday.com/view/more-than-skin-deep-improving-physical- appearanceafter-cancer. Acesso em: 09 ago. 2020

MORELLE, Alessandra. Mantenha a autoestima durante o tratamento do câncer. 2018. Disponível em: https://alessandramorelle.com.br/autoestima-durante-o- tratamento-docancer/. Acesso em: 16 dez. 2020.

MOTA, Dryelle Feitosa et al. COSMÉTICOS AÇÃO E REAÇÃO. Revista Interfaces: Saúde, Humanas e Tecnologia., Juazeiro do Norte, v. 2, n. 4, p. 1-5, 16 maio 2014. Disponível em:https://interfaces.leaosampaio.edu.br/index.php/revista-interfaces/article/view/67. Acesso em: 28 ago. 2020.

NETEMEYER, Richard G.; BURTON, Scot; LICHTENSTEIN, Donald R.. Trait Aspects of Vanity: measurement and relevance to consumer behavior. Journal Of Consumer Research, [S.L.], v. 21, n. 4, p. 612-626, mar. 1995. Oxford University Press (OUP). http://dx.doi.org/10.1086/209422.

OLIVEIRA, Lays Aylanne Borges; ARAÚJO, Keila Gomes de Souza; FERNANDES, Yanne Maria de Souza. Do cosmético a camuflagem. 2012. 20 f. TCC (Graduação) - Curso de Farmácia, Faculdade União de Goyazes, Trindade, 2012. Disponível em: http://www.fug.edu.br/repositorio/2012- 2/Farmacia/DO\%20COSM\%C3\%89TICO\%20A\% 
20CAMUFLAGEM.pdf. Acesso em: 22 dez. 2020.

OPAS. OPAS/OMS apoia governos no objetivo de fortalecer e promover a saúde mental da população. Disponível em: https://www.paho.org/bra/index.php?option=com_content\&view =article\&id=5263:opas-oms-apoia-governos-no-objetivo-de-fortalecer-e-promover-a-audemental-da- populacao\&ltemid=839. Acesso em: 08 jul. 2020.

ROARK, Louanne. Look Good ... Feel Better Helps People Battling Cancer Help Themselves. Journal Of Oncology Practice, [S.L.], v. 4, n. 5, p. 262-262, set. 2008. American Society of Clinical Oncology (ASCO). http://dx.doi.org/10.1200/jop.0854502.

SAKS, Flavia do Canto. Busca Booleana: Teoria E Prática. 2005. 61 f. TCC (Graduação) Curso de Gestão da Informação, Universidade Federal do Paraná, Curitiba, 2005.

SILVA, Luana Alexandrino Rodrigues da. O impacto do uso de cosméticos no bem-estar de pacientes em tratamento oncológico. 2018. 17 f. TCC (Graduação) - Curso de Farmácia, Universidade Federal de São Paulo, Diadema, 2018.

STREHLAU, V. I; CLARO, D. P; NETO, S. A. L. Espelho, espelho meu, existe alguém mais vaidosa do que eu? A vaidade feminina e sua influência na autoestima e no consumo de procedimentos estéticos. IN: ENCONTRO DE MARKETING DA ASSOCIAÇÃO NACIONAL DE PÓS-GRADUAÇÃO E PESQUISA EM ADMINISTRAÇÃO, 4, 2010, Florianópolis. Anais. Florianopolis: EMA, 2010

SURVEY, Harris. Topline Key Messages and Findings. [S.I]: Look Good Feel Better, 2012. Disponível em: https://lookgoodfeelbetter.org/wp-content/uploads/pdf/PDF-\%202012\%20 LGFB\%20WEek\%20Harris\%20Topline\%20FINAL\%204-20-12.pdf. Acesso em: 08 out. 2020.

TAGGART, Linda; OZOLINS, Laura; HARDIE, Heather; NYHOF-YOUNG, Joyce. Look Good Feel Better Workshops: a .:big lift:: for women with cancer. Journal Of Cancer Education, [S.L.], v. 24, n. 2, p. 94-99, abr. 2009. Springer Science and Business Media LLC. http://dx.doi.org/10.1080/08858190802664594.

VIGGIAN, Claudio. Um Projeto Que Transforma Vidas! 2017. Disponível em: 
https://institutoabihpec.org.br/de-bem-com-voce/um-projeto-que-transforma-vidas/. Acesso em: 26 set. 2020.

VOSGERAU, Dilmeire Sant'Anna Ramos; ROMANOWSKI, Joana Paulin. Estudos de revisão: implicações conceituais e metodológicas. Rev. Diálogo Educ, Curitiba, v. 14, n. 41, p. 165-189, jan. 2014.

WIRE, Business Oferecer consistentemente uma experiência benéfica: os dados da pesquisa global Look Good Feel Better mostram o impacto do programa entre várias culturas e continentes. 2019. Disponível em: https://www.businesswire.com/news/home/ $20200204005087 /$ pt/. Acesso em: 26 set. 2020.

WIRE, Business. O custo incalculável do câncer: mudanças na aparência devido ao impacto do tratamento do câncer nos relacionamentos pessoais de acordo com a nova pesquisa nacional de mulheres com câncer. 2012. Disponível em: https://www.businesswire.com/ news/home/20120424005265/en/Untold-Cost-Cancer-Appearance-Due-Cancer-reatment. Acesso em: 08 out. 2020.

Autor para correspondência:

Mônica Santos

Email: monica.quimica@yahoo.com.br

Faculdade de Tecnologia de Diadema Luigi Papaiz.

Recebido: 05/02/2021 Aceito: 27/03/2021 